\title{
Geometry and Control of Three-Wave Interactions
}

\author{
Mark S. Alber \\ Department of Mathematics \\ University of Notre Dame \\ Notre Dame, IN 46556 \\ email: Mark.S.Alber.1@nd.edu \\ Gregory G. Luther \\ Engineering Sciences and Applied Mathematics Department \\ McCormick School of Engineering and Applied Science \\ Northwestern University \\ 2145 Sheridan Road \\ Evanston, Il 60208-3125. \\ email: ggluther@nwu.edu \\ Jerrold E. Marsden \\ Control and Dynamical Systems 107-81 \\ Caltech, \\ Pasadena, CA 91125 \\ email: marsden@cds.caltech.edu \\ Jonathan M. Robbins \\ The Basic Research Institute in the Mathematical Sciences (BRIMS) \\ Hewlett-Packard Laboratories \\ Filton Road, Stoke Gifford \\ Bristol BS12 6QZ, UK \\ and \\ School of Mathematics \\ University Walk, Bristol BS8 1TW UK \\ email: j.robbins@bristol.ac.uk
}

October 13, 1998

\begin{abstract}
The integrable structure of the three-wave equations is discussed in the setting of geometric mechanics. Lie-Poisson structures with quadratic Hamiltonian are associated with the three-wave equations through the Lie algebras $\mathfrak{s u}(3)$ and $\mathfrak{s u}(2,1)$. A second structure having cubic Hamiltonian is shown to be compatible. The analogy between this system and the rigid-body or Euler equations is discussed. Poisson reduction is performed using the method of invariants and geometric phases associated with the reconstruction are calculated. We show that using piecewise continuous controls, the transfer of energy among three
\end{abstract}


waves can be controlled. The so called quasi-phase-matching control strategy, which is used in a host of nonlinear optical devices to convert laser light from one frequency to another, is described in this context. Finally, we discuss the connection between piecewise constant controls and billiards.

\section{Contents}

1 Introduction $\quad 2$

2 The Canonical Hamiltonian Structure. 5

$\begin{array}{lll}3 & \text { Poisson Reduction } & 7\end{array}$

4 Geometric Control of Three-wave Interactions 11

5 Projection of the Reduced Phase Space and Billiards 14

6 Reconstruction and Phases $\quad 17$

$\begin{array}{llr}7 & \text { The Lie-Poisson Formulation } & 18\end{array}$

8 Connections between the Two Hamiltonian Structures 23

9 Discussion $\quad 27$

\section{Introduction}

The three-wave equations model the dynamics of the amplitudes of three light-waveenvelopes as they interact through the quadratic response of a nonlinear optical material. They are also used to model resonant wave interactions in fluids and plasmas. During the asymptotic reduction from Maxwell's equations or another set of primitive equations, one assumes that the wave vectors and frequencies of the three waves are nearly resonant in the sense that $\mathbf{k}_{1}\left(\omega_{1}\right) \simeq \mathbf{k}_{2}\left(\omega_{2}\right)-\mathbf{k}_{3}\left(\omega_{3}\right)$, and $\omega_{1} \simeq$ $\omega_{2}-\omega_{3}$ for a decay process or the matching conditions $\mathbf{k}_{1}\left(\omega_{1}\right) \simeq-\mathbf{k}_{2}\left(\omega_{2}\right)-\mathbf{k}_{3}\left(\omega_{3}\right)$, and $\omega_{1} \simeq-\omega_{2}-\omega_{3}$ for an explosive process. Since the wave vectors are functions of the frequencies, a given set of frequencies may not satisfy the resonance condition for both frequencies and wave vectors. This restrictive condition is overcome in order to enhance or suppress the conversion of light from one frequency to another by controlling the three-wave interaction.

The quadratic three-wave system of ordinary differential equations (representing 
the dynamics of traveling waves for the three-wave PDE) is

$$
\begin{aligned}
\frac{d q_{1}}{d t} & =i(\Delta k) q_{1}+i s_{1} \gamma_{1} q_{2} \bar{q}_{3} \\
\frac{d q_{2}}{d t} & =i(\Delta k) q_{2}+i s_{2} \gamma_{2} q_{1} q_{3} \\
\frac{d q_{3}}{d t} & =i(\Delta k) q_{3}+i s_{3} \gamma_{3} \bar{q}_{1} q_{2} .
\end{aligned}
$$

We now explain the notation. First of all, each $q_{i} \in \mathbb{C}$, so this is a parameter dependent system of ordinary differential equations on complex three space, $\mathbb{C}^{3}$. We have chosen the evolution variable to be $t$ here; in an optical wave interaction this often corresponds to the distance the waves propagate, usually denoted $z$. The term $\Delta k$ is the amount by which the wave vectors of the three wave envelopes fail to be in resonance for a given set of three frequencies. Assuming that the waves propagate nearly collinearly in the $z$-direction, $\Delta k$ is the $z$-component of the mismatch among the wave vectors, so $\Delta k=\left(\mathbf{k}_{1}-\mathbf{k}_{2}+\mathbf{k}_{3}\right) \cdot \hat{\mathbf{z}}$, where $\hat{\mathbf{z}}$ is a unit vector in the $z$-direction.

The $\gamma_{i}$ are nonzero real numbers such that $\gamma_{1}+\gamma_{2}+\gamma_{3}=0$. They measure the strength of the nonlinear coupling among the waves. For a fixed choice of the $\gamma_{j}$, the choice of signs, determined by $\left(s_{1}, s_{2}, s_{3}\right)$ distinguishes between three decay interactions which have bounded solutions in time and an explosive interaction which has solutions that blow up in finite time. Choosing $\gamma_{1}, \gamma_{3}>0$ and $\gamma_{2}<0$, the decay systems are obtained by choosing $\left(s_{1}, s_{2}, s_{3}\right)$ to be $(1,-1,1),(1,-1,-1)$ or $(-1,-1,1)$. The explosive system is obtained by choosing $(-1,-1,-1)$.

One of the main objectives of this paper is to discuss some control theoretic aspects of these equations. To do so, we shall make use of symmetry and reduction appropriate to these equations. Thus, we first shall review some aspects of this symmetry and reduction from Alber, Luther, Marsden and Robbins [1998a].

Reduction theory for these equations can be viewed in two useful ways. First, by means of invariants and second, by viewing the equations as Lie-Poisson equations for the groups $\mathrm{SU}(3)$ and $\mathrm{SU}(2,1)$. More generally, the structure of the $n$-wave interaction is related to the family of Lie groups $\mathrm{SU}(n)$ and $\mathrm{SU}(p, q)$ and their Lie algebras $\mathfrak{s u}(n)$ and $\mathfrak{s u}(p, q)$. We shall explain both of these views of the equations.

Basic wave interactions of this kind are fundamental in the understanding and analysis of a variety of phenomena including patterns, symmetry induced instabilities, the Benjamin-Feir instability and many others. The three-wave equations are closely related to the equations governing coupled harmonic oscillators, tops, and the rigid body. This is understood by realizing that the three-wave equations are the complex equations for a resonant three degree of freedom Hamiltonian system.

There is a strong association between the three-wave system and the Euler equations for the motion of a free rigid body. The Euler equations are realized on a real subspace of the three-wave equations (see for instance, Guckenheimer and Mahalov [1992]). Below we write the three-wave equations as an Euler equation associated with groups $\mathrm{SU}(3)$ or $\mathrm{SU}(2,1)$. In this context it is clear that $\mathrm{SO}(3)$ as a real subgroup produces the Euler equations for an appropriate rigid body. We develop 
this analogy further between the real and complex equations, and in doing so the connection to the $n$-component Euler equations is made (Manakov [1976]).

The integrable Hamiltonian structure of the three-wave equations is of course well known; here we explore it from a somewhat novel point of view, namely that of compatibility between the canonical Hamiltonian and the Lie-Poisson structures.

Using the Lagrangian point of view, the three-wave system can be realized as a set of Euler-Poincaré equations, while from the Hamiltonian point of view, the equations are Lie-Poisson. One of the three-wave decay systems is Lie-Poisson for the Lie algebra $\mathfrak{s u}(3)$, while two of the decay systems and the explosive threewave system are Lie-Poisson for $\mathfrak{s u}(2,1)$. Using the method of translation of the argument, two compatible Hamiltonian structures are obtained. One is the canonical Hamiltonian structure embedded in $\mathfrak{s u}(3)$ (respectively $\mathfrak{s u}(2,1)$ ) in a way we will explain, and it has a cubic Hamiltonian. The other is non-canonical having the standard left invariant Lie-Poisson bracket and it has a quadratic Hamiltonian. These two Poisson brackets lead to a recursion relation that is expressed in terms of Lie brackets, and this recursion relation is the same one that is found using the Lax pair approach.

Solutions for the integrable three-wave equations and other similar systems are well known. In our approach below, they are reduced and integrated using a pair of $S^{1}$ actions, the canonical Hamiltonian structure and the technique of invariants. In solving the reconstruction problem, phase formulas analogous to those obtained for the rigid body (Montgomery [1991]) are obtained. These formulas give the value of the phase shifts that accompany the periodic exchange of wave action in resonant wave systems (see for example McKinstrie [1988], McKinstrie and Luther [1988], McKinstrie and Cao [1993], Alber, Luther and Marsden [1997], Alber, Luther, Marsden and Robbins [1998a]).

Kummer [1990] treated reduction for the $n$-degree of freedom Hamiltonian with resonances. Our approach differs somewhat in that we choose the invariant coordinates for the reduced phase space in such a way that a family of pinched spheres lie along a vertical axis. These coordinates also produce Hamiltonians that are planes depending on only two of the reduced coordinates. The choice of coordinates for the reduced phase space described below provide a particularly simple geometrical understanding for controlling the dynamics of the waves.

The reduced phase space is a useful setting in which to understand and analyze control strategies for three-wave mixing in nonlinear optics. Armstrong, Bloembergen, Ducuing, and Pershan [1962] proposed that the flow of energy among interacting light waves could be controlled by modulating the sign of the quadratic nonlinear coupling constants. These ideas have been particularly useful for converting light to its second-harmonic frequency in optical waveguides (Fejer, Magel, Jundt, and Byer [1992]) where the quadratic coefficients are modulated along the direction of propagation of the waves by periodically reorienting the molecular structure of the host material. We show below that this so called quasi-phase-matching technique which is used to convert the light from one frequency to another (Armstrong, Bloembergen, Ducuing, and Pershan [1962], Fejer, Magel, Jundt, and Byer [1992]) has a simple geometric description. This geometric approach also provides a com- 
plete description of possible control strategies for three interacting light waves and has been extended to treat more waves.

The general picture developed here is useful for many other purposes, including the understanding of polarization dynamics as in David, Holm, and Tratnik [1989] or Akhmediev and Ankiewicz [1997], and perturbations of Hamiltonian normal forms as in the work of Knobloch, Mahalov, and Marsden [1994], Kirk, Marsden, and Silber [1996], and Haller and Wiggins [1996]. Notice that this geometric description generalizes the construction of the Poincaré sphere that is typically used in the analysis of polarization dynamics (see Born and Wolf [1980] and David, Holm, and Tratnik [1989].)

In this study of piecewise constant controls on the reduced phase space of the three-wave equations, a series of generalized billiard problems arises. The locus of points where piecewise controls are switched between two states, form a boundary on the three-wave surface at which a generalized reflection can be defined. In certain cases, these billiards project in a simple and natural way into a plane. This is an interesting example of the connection between nonlinear controls and billiards. A discussion of billiards in the context of the reduced three-wave equations is included in $\S 5$.

\section{The Canonical Hamiltonian Structure.}

We shall first describe a canonical Poisson structure using a $\gamma_{i}$-weighted canonical Poisson bracket on $\mathbb{C}^{3}$. This bracket has the real and imaginary parts of each complex dynamical variable $q_{i}$ as conjugate variables. The Hamiltonian for the three-wave equations is cubic in this setting.

The Canonical Symplectic and Poisson Structure. Writing $q_{k}=x_{k}+i y_{k}$ and treating $x_{k}$ and $y_{k}$ as conjugate variables, the (scaled) canonical Poisson bracket is given by

$$
\{F, G\}=\sum_{k=1}^{3} s_{k} \gamma_{k}\left(\frac{\partial F}{\partial x_{k}} \frac{\partial G}{\partial y_{k}}-\frac{\partial G}{\partial x_{k}} \frac{\partial F}{\partial y_{k}}\right) .
$$

In matrix notation, this reads

$$
\{F, K\}=(\nabla F) \mathbb{J}(\nabla K),
$$

where the gradients are standard gradients in $\mathbb{R}^{6}$ (with the variables ordered as $\left.\left(x_{1}, x_{2}, x_{3}, y_{1}, y_{2}, y_{3}\right)\right)$ and where

$$
\mathbb{J}=\left(\begin{array}{cc}
0 & \Gamma \\
-\Gamma & 0
\end{array}\right)
$$

in which $\Gamma$ is the $3 \times 3$ matrix with $s_{k} \gamma_{k}$ on the diagonal and zeros elsewhere. 
This bracket may be written in complex notation as

$$
\{F, G\}=-2 i \sum_{k=1}^{3} s_{k} \gamma_{k}\left(\frac{\partial F}{\partial q^{k}} \frac{\partial G}{\partial \bar{q}_{k}}-\frac{\partial G}{\partial q^{k}} \frac{\partial F}{\partial \bar{q}_{k}}\right) .
$$

The corresponding symplectic structure is given as follows:

$$
\omega\left(\left(z_{1}, z_{2}, z_{3}\right),\left(w_{1}, w_{2}, w_{3}\right)\right)=-\sum_{k=1}^{3} \frac{1}{s_{k} \gamma_{k}} \operatorname{Im}\left(z_{k} \bar{w}_{k}\right) .
$$

The Hamiltonian. The Hamiltonian for the three-wave interaction is

$$
H_{3}=-\frac{1}{2} \Delta k \sum_{k=1}^{3} \frac{\left|q_{k}\right|^{2}}{s_{k} \gamma_{k}}-\frac{1}{2}\left(\bar{q}_{1} q_{2} \bar{q}_{3}+q_{1} \bar{q}_{2} q_{3}\right) .
$$

Hamilton's equations for a Hamiltonian $H$ are the standard ones written in Poisson bracket form as $\dot{F}=\{F, H\}$, or equivalently,

$$
\frac{d q_{k}}{d t}=\left\{q_{k}, H\right\}
$$

It is straightforward to check that Hamilton's equations in our case are given in complex notation by

$$
\frac{d q_{k}}{d t}=-2 i s_{k} \gamma_{k} \frac{\partial H}{\partial \bar{q}_{k}}
$$

One checks that Hamilton's equations with $H=H_{3}$ coincide with (1.1)-(1.3).

This computation shows the following standard result.

Proposition 2.1 With the preceding Hamiltonian $\mathrm{H}_{3}$ and the symplectic or equivalently the Poisson structure given above, Hamilton's equations are given by the three-wave equations (1.1)-(1.3).

Integrals of Motion. In addition to $H_{3}$ itself, one can easily check that the following quantities are constants of motion:

$$
\begin{aligned}
& K_{1}=\frac{1}{2}\left(\frac{\left|q_{1}\right|^{2}}{s_{1} \gamma_{1}}+\frac{\left|q_{2}\right|^{2}}{s_{2} \gamma_{2}}\right), \\
& K_{2}=\frac{1}{2}\left(\frac{\left|q_{2}\right|^{2}}{s_{2} \gamma_{2}}+\frac{\left|q_{3}\right|^{2}}{s_{3} \gamma_{3}}\right), \\
& K_{3}=\frac{1}{2}\left(\frac{\left|q_{1}\right|^{2}}{s_{1} \gamma_{1}}-\frac{\left|q_{3}\right|^{2}}{s_{3} \gamma_{3}}\right) .
\end{aligned}
$$

These are often referred to as the Manley-Rowe relations. The Hamiltonian with any two of the $K_{j}$ are checked to be a complete and independent set of conserved 
quantities in involution (the $K_{j}$ clearly give only two independent invariants since $\left.K_{1}-K_{2}=K_{3}\right)$. One concludes that the system is integrable the sense of LiouvilleArnol'd (see, e.g., Arnol'd [1989] or Abraham and Marsden [1978]).

One can reinterpret the Manley-Rowe relations in terms of momentum maps in the following way.

Proposition 2.2 The vector function $\left(K_{1}, K_{2}, K_{3}\right)$ is the momentum map for the following symplectic action of $T^{3}=S^{1} \times S^{1} \times S^{1}$ :

$$
\begin{aligned}
& \left(q_{1}, q_{2}, q_{3}\right) \mapsto\left(q_{1} \exp (-i \gamma), q_{2} \exp (-i \gamma), q_{3}\right) \\
& \left(q_{1}, q_{2}, q_{3}\right) \mapsto\left(q_{1}, q_{2} \exp (-i \gamma), q_{3} \exp (-i \gamma)\right) \\
& \left(q_{1}, q_{2}, q_{3}\right) \mapsto\left(q_{1} \exp (-i \gamma), q_{2}, q_{3} \exp (i \gamma)\right)
\end{aligned}
$$

Any combination of two of these actions is generated by the third reflecting the fact that the $K_{j}$ are linearly dependent. Another way of saying this is that the group action by $T^{3}$ is really captured by the action of $T^{2}$.

\section{Poisson Reduction}

Poisson reduction may now be performed on the three-wave Hamiltonian system using the $S^{1}$ symmetries associated with the momentum maps $K_{k}$. The Poisson reduction process considers the quotient bundle $\mathbb{C}^{3} \rightarrow \mathbb{C}^{3} / T^{2}$ and puts the unique Poisson structure on the quotient that makes the projection to the quotient a Poisson map (see Marsden and Ratiu [1998], Chapter 10). The symplectic leaves in the quotient $\mathbb{C}^{3} / T^{2}$, which are the symplectic reduced spaces, will be analyzed using the method of invariants.

Invariants as Coordinates for Three-wave Reduction. Invariants for the $T^{2}$ action (i.e., functions invariant under the $T^{2}$ action) are:

$$
\begin{aligned}
X+i Y & =q_{1} \bar{q}_{2} q_{3}, \\
Z_{1} & =\left|q_{1}\right|^{2}-\left|q_{2}\right|^{2}, \\
Z_{2} & =\left|q_{2}\right|^{2}-\left|q_{3}\right|^{2} .
\end{aligned}
$$

These quantities provide coordinates for the four (real)-dimensional orbit space $\mathbb{C}^{3} / T^{2}$. The coordinates, $X, Y, Z_{1}$ and $Z_{2}$ are Hopf-like variables (see, e.g., Cushman and Rod [1982]) and they generalize the well known Stokes parameters (see, e.g., Born and Wolf [1980]) to resonant interactions for systems with more than two complex components.

Reduced Three-wave Surfaces. The following identity holds amongst the invariants and the conserved quantities:

$$
X^{2}+Y^{2}=\kappa_{4}\left(2 s_{2} \gamma_{2} K_{1}+Z_{1}\right)\left(2 s_{3} \gamma_{3} K_{2}+Z_{2}\right)\left(2 s_{2} \gamma_{2} K_{2}-Z_{2}\right)
$$


where

$$
\kappa_{4}=\frac{s_{1} \gamma_{1} s_{2} \gamma_{2} s_{3} \gamma_{3}}{\left(s_{1} \gamma_{1}+s_{2} \gamma_{2}\right)\left(s_{2} \gamma_{2}+s_{3} \gamma_{3}\right)^{2}} .
$$

Trajectories in these reduced coordinates lie on the set defined by this relation. Using the conservation laws $K_{k}$ and the definitions of $Z_{1}$ and $Z_{2}$, one obtains a second relation between $K_{1}, K_{2}$ and $Z_{1}, Z_{2}$ and either of the coordinates $Z_{j}$ may then be removed, thereby reducing the number of real dimensions to three. This procedure allows one to realize the reduced trajectories as lying on the following invariant set in $\mathbb{R}^{3}$ :

$$
X^{2}+Y^{2}=\kappa_{3}\left(\delta-Z_{2}\right)\left(2 s_{3} \gamma_{3} K_{2}+Z_{2}\right)\left(2 s_{2} \gamma_{2} K_{2}-Z_{2}\right),
$$

where

$$
\kappa_{3}=\frac{s_{1} \gamma_{1} s_{2} \gamma_{2} s_{3} \gamma_{3}}{\left(s_{2} \gamma_{2}+s_{3} \gamma_{3}\right)^{3}}
$$

and

$$
\delta=2 s_{2} \gamma_{2} K_{1}+2 s_{3} \gamma_{3}\left(K_{1}-K_{2}\right) .
$$

This relation defines a two dimensional, perhaps singular, surface (also called an orbifold as in Sjamaar and Lerman [1991]) in $\left(X, Y, Z_{2}\right)$ space, with $Z_{1}$ determined by the values of these invariants and the conserved quantities; thus, it may also be thought of as a surface in $\left(X, Y, Z_{1}, Z_{2}\right)$. The relations between the invariants and the conserved quantities may imply inequalities for, say, $Z_{2}$; for example, these are useful in determining when the corresponding surface is compact. A sample of one of these surfaces is plotted in Fig. 3.1. These surfaces will be called the three-wave surfaces below.

Reduced Three-wave Equations. Any trajectory of the original equations defines a curve on each three-wave surface, in which the $K_{j}$ are set to constants. These three-wave surfaces are the symplectic leaves in the four-dimensional Poisson space $\mathbb{C}^{3} / T^{2}$ having coordinates $\left(X, Y, Z_{1}, Z_{2}\right)$.

The original equations define a dynamical system in the Poisson reduced space and on the symplectic leaves as well. Using these new coordinates, the Poisson bracket and the Hamiltonian are reduced directly. The reduced Hamiltonian is

$$
H_{r}=-X-\frac{\Delta k}{2\left(s_{2} \gamma_{2}+s_{3} \gamma_{3}\right)}\left(2\left(s_{2} \gamma_{2}+s_{3} \gamma_{3}\right) K_{1}+2 s_{2} \gamma_{2} K_{2}-Z_{2}\right) .
$$

Using the reduced Poisson brackets and the variables $\left(X, Y, Z_{2}\right)$, the Hamiltonian $H_{r}$ produces the following reduced equations of motion

$$
\begin{aligned}
\frac{d X}{d t} & =-\Delta k Y, \\
\frac{d Y}{d t} & =\Delta k X+\frac{\partial \phi}{\partial Z_{2}}, \\
\frac{d Z_{2}}{d t} & =-2\left(s_{2} \gamma_{2}+s_{3} \gamma_{3}\right) Y,
\end{aligned}
$$




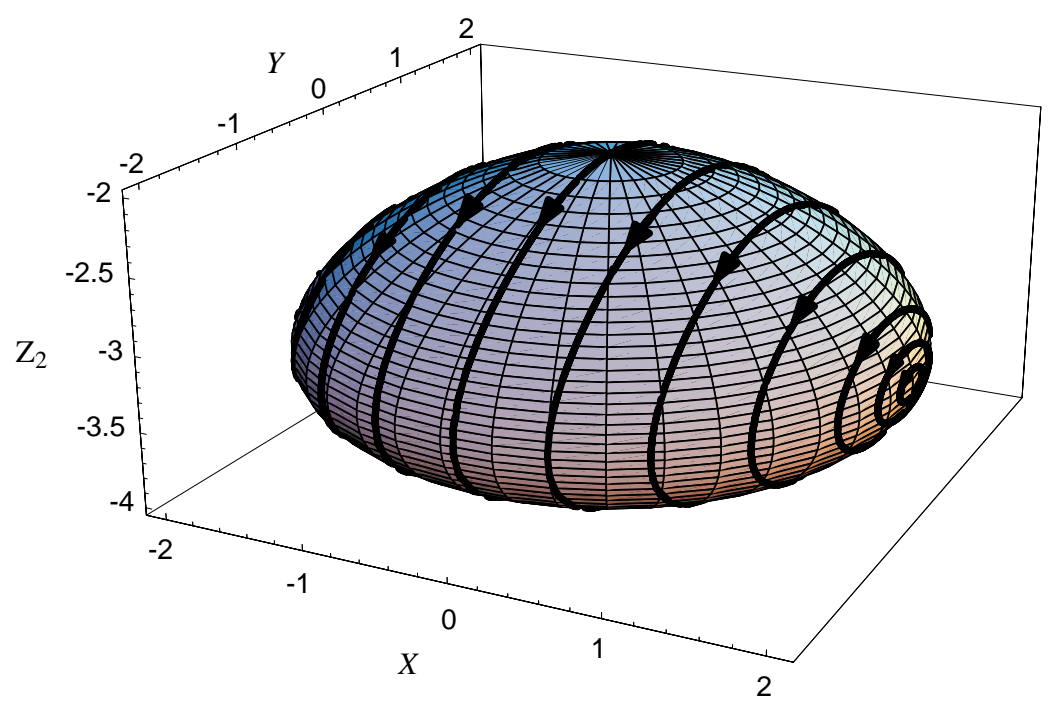

Figure 3.1: A three-wave surface is drawn in $\left(X, Y, Z_{2}\right)$ coordinates for the decay interaction. Trajectories are also drawn showing the phase space of the reduced three-wave equations on the three-wave surface when $\left(s_{1}, s_{2}, s_{3}\right)=(1,1,1),\left(\gamma_{1}, \gamma_{2}, \gamma_{3}\right)=(1,1,-2)$, and $\left(K_{1}, K_{2}\right)=(1,-1 / 2)$.

where the dynamical invariant $\phi$ is defined by

$$
\begin{aligned}
\phi= & \left(s_{2} \gamma_{2}+s_{3} \gamma_{3}\right)\left[\left(X^{2}+Y^{2}\right)\right. \\
& \left.-\kappa_{3}\left(\delta-Z_{2}\right)\left(2 s_{3} \gamma_{3} K_{2}+Z_{2}\right)\left(2 s_{2} \gamma_{2} K_{2}-Z_{2}\right)\right] .
\end{aligned}
$$

Following Kummer [1975, 1990], the reduced equations may be written as $\dot{F}=$ $\left\{F, H_{r}\right\}$ for the Poisson bracket

$$
\{F, G\}=\nabla \phi \cdot(\nabla F \times \nabla G) .
$$

The Poisson structure on $\mathbb{C}^{3}$ drops to a Poisson structure on $\left(X, Y, Z_{1}, Z_{2}\right)$-space and this in turn induces the Poisson structure above. Correspondingly, the symplectic structure drops to one on each three-wave surface - this is an example of the general procedure of symplectic reduction (see Marsden and Weinstein [1974]).

The three-wave surfaces may have singularities - this is because the group action is not free; in this case it is a fairly simple "orbifold" singularity. For the three-wave system a singular point appears on the three-wave surface when $\left|q_{1}\right|^{2} /\left(s_{1} \gamma_{1}\right)=$ $\left|q_{3}\right|^{2} /\left(s_{3} \gamma_{3}\right)$ or equivalently $K_{1}=K_{2}$ and $K_{3}=0$. In this case two of the roots of the cubic polynomial in $\phi$ come together. From the geometry, it is also clear that a homoclinic orbit passes through such a singular point.

Equations (3.11) show that $H_{r}$ and $\phi$ are constants of the motion. Expressed in terms of the wave amplitudes $q_{j}, \phi\left(X, Y, Z_{2}\right)$ vanishes identically. Thus the reduced dynamics is confined to the three-wave surfaces defined by $\phi\left(X, Y, Z_{2}\right)=0$. Trajectories of the reduced equations are the curves produced by intersecting the 
three-wave surfaces with level sets of $H_{r}$. Since $H_{r}$ is linear, its level sets are the planes

$$
Z_{2}=m X+\left(\gamma_{2}+\gamma_{3}\right)\left(K_{1}+\frac{2 H_{r}}{\Delta k}\right)+\gamma_{2} K_{2}
$$

where $m=2\left(\gamma_{2}+\gamma_{3}\right) / \Delta k$.

A reduced phase space is plotted in Fig. 3.2. For the fixed point set a similar surface is obtained, but a singular point associated with a homoclinic trajectory appears along $(X, Y)=(0,0)$.

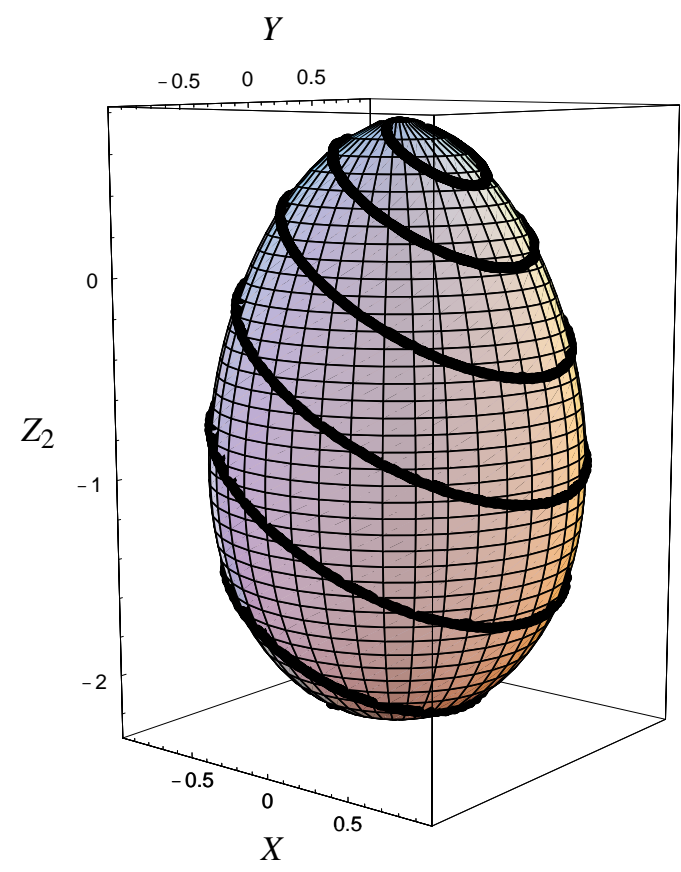

Figure 3.2: A reduced three-wave phase space on a three-wave surface. Here the mismatch is not zero, and $|m|=3 / 5$, where $\left(\gamma_{1}, \gamma_{2}, \gamma_{3}\right)=(-1,-2,-1), \Delta k=10.0$ and the point $\left(q_{1}(0), q_{2}(0), q_{3}(0)\right)=(1.0,0.05,1.5)$ is used to fix $K_{1}, K_{2}$ and $H$.

As the slope, $m$, of the Hamiltonian planes varies, the qualitative nature of the dynamics changes. When $m$ is small, the Hamiltonian planes and therefore orbits on the three-wave surfaces are nearly horizontal. For fixed $\gamma_{j}, m$ is small far from phase matching $(\Delta k=0)$. Here, the linear oscillation captured in Eqs. (3.7) and (3.8) dominates, so the dynamics is well approximated by a driven harmonic oscillator with oscillation period $2 \pi / \Delta k$.

Since the orbits are nearly horizontal over the entire three-wave surface, this approximation is valid over most of the reduced phase space. It breaks down only when a homoclinic orbit connected to the singular point mentioned above is present. For small $m$ this region occupies a small portion of the three-wave surface. For large $m$ the orbits are nearly vertical and the nonlinear oscillation captured in Eqs. 
(3.8) and (3.9) dominates. The two fixed points move from the top and bottom of the three-wave surface when $m=0$ to the sides along $Y=0$ when $m=\infty$ and are positioned at points where the Hamiltonian plane is tangent to the three-wave surface.

In applications the goal is often to produce the largest amount of conversion among the waves. This means making the largest vertical excursion on the threewave surface. When $\Delta k=0$, the orbit passing through $(X, Y)=(0,0)$ produces maximum conversion. When the singularity is present this is the homoclinic orbit, where $H_{r}=0$. For intermediate values of $m$ a trajectory has components of both the horizontal or linear oscillation and the vertical or nonlinear oscillation. Here the orbit with the largest variation in $Z_{2}$ produces maximum conversion. In some applications the goal is to produce the largest phase shift with a minimum amount of conversion.

\section{Geometric Control of Three-wave Interactions}

In many applications the goal is to convert as much light at frequency $\omega_{1}$ into light at the frequencies $\omega_{2}$ and $\omega_{3}$. Since $\left|q_{1}\right|$ is large compared to either $\left|q_{2}\right|$ or $\left|q_{3}\right|$ at the bottom of the three-wave surface, most of the light is at frequency $\omega_{1}$ there. Near the top the light at $\omega_{1}$ has been converted into the two other waves. Notice that when $\Delta k=0$ a trajectory at the bottom of the three-wave surface connects to the top. This would seem to be the most desirable situation if the goal is to produce the maximum conversion for a given three-wave interaction. Unfortunately this condition is typically difficult to achieve with available materials and device constraints. More often $\Delta k \gg \gamma_{j}$ for each $j$ and the orbits are nearly horizontal. Thus the amount of wave energy or action converted during each orbit is relatively small. This problem has been circumvented by introducing a piecewise constant control (see Armstrong, Bloembergen, Ducuing, and Pershan [1962] and Fejer, Magel, Jundt, and Byer [1992]). The key point is that reversing the signs of the $\gamma_{j}$ leaves the three-wave surfaces invariant while changing the slope of the Hamiltonian planes. Since this change in signs only changes the direction of increase in the equation for $Z_{2}$ in (3.7)-(3.9), a $C^{0}$ trajectory that spirals up the three-wave surface can be generated. Just as the wave interaction saturates and light begins to convert back to frequency $\omega_{1}$, the direction of conversion is reversed by inverting the sign of $\gamma_{j}$. The light-wave energy or action then continues to flow into the waves at frequencies $\omega_{2}$ and $\omega_{3}$. This control strategy is called quasi-phase-matching in nonlinear optics.

To reach the top of the three-wave surface quasi-phase-matching is performed by alternating the signs of the $\gamma_{j}$ at every half-period of the oscillation cycle. In general, the half-periods will depend on $Z_{2}$. For small $m$ this dependence is weak, so in practice the period for the control is approximated by half the linear oscillation period, i.e., the coherence length, defined as $l_{c}=\pi / \Delta k$.

Quasi-phase-matching is now described geometrically on the three-wave surfaces. The fact that the substitution $\gamma_{j} \rightarrow-\gamma_{j}$ leaves the three-wave surfaces invariant but reverses the slope of the Hamiltonian planes leads to the following geometrical construction for quasi-phase-matching trajectories: they are obtained by concatenating 
intersections of the three-wave surface with Hamiltonian planes of alternating slope. In the optimal case, the sign changes along $Y=0$, at a point of maximal $Z_{2}$ on one segment and minimal $Z_{2}$ on the next. In Fig. 4.1 a quasi-phase-matching trajectory is plotted on a three-wave surface.
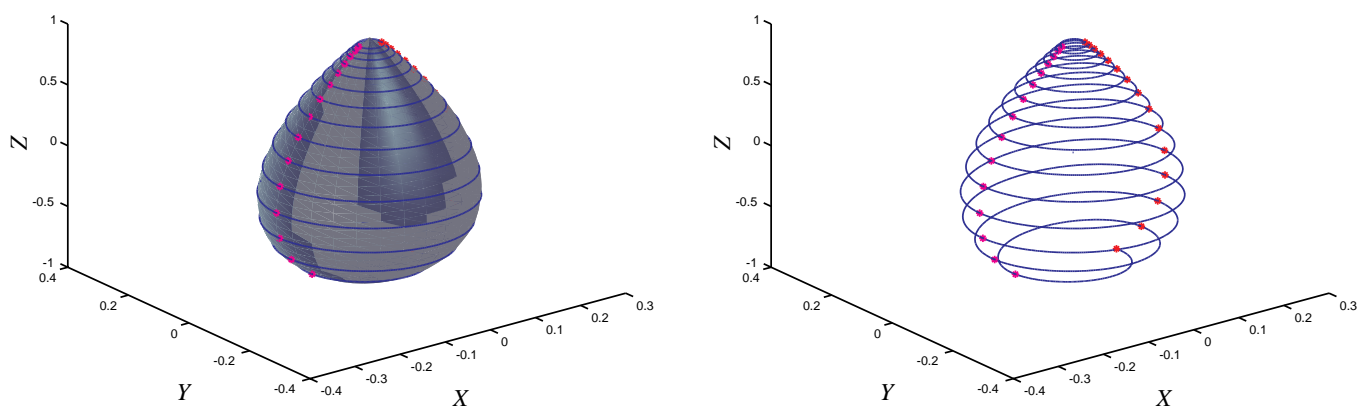

Figure 4.1: A composite trajectory with 30 segments of length $l_{c}=\pi / \Delta k$ for the quasiphase-matched control of the three-wave system. Here, $|m|=0.3$, where $\left(\gamma_{1}, \gamma_{2}, \gamma_{3}\right)=$ $(-1,-2,-1), \Delta k=30.0$ and $\left(q_{1}(0), q_{2}(0), q_{3}(0)\right)=(0.1,1.0,0.2)$ is used to fix $K_{1}, K_{2}$ and $H$.

The curve in this figure was generated numerically from Eqs. (1.1)-(1.3) by alternating the signs of the $\gamma_{j}$ after steps of size $l_{c}$. It spirals up the three-wave surface towards larger values of $Z_{2}$ as more light is converted. The initial data is on $Y=0$ with $X>0$ since $m=-0.3$ for $\left(\gamma_{1}, \gamma_{2}, \gamma_{3}\right)=(-1,-2,-1)$ and $\Delta k=30$. Note that excellent conversion efficiency is achieved after 30 layers in this example even though $l_{c}$ is used. In a typical optical device the value of $\Delta k$ may be much larger and as many as 500 to 1000 layers may be used.

The nonlinear component of the oscillation contributes a small shift to the linear period, $2 l_{c}$. This shift leads to the eventual saturation of the quasi-phase-matching conversion. As $m$ increases, the linear period is an increasingly poor approximation to the actual oscillation period and the quasi-phase-matching conversion saturates after only a few steps of length $l_{c}$. At these larger values of $m$, the signs of the quadratic coefficients must be alternated at half the nonlinear period to obtain the most efficient quasi-phase-matching conversion. To produce maximum secondharmonic conversion, the initial data are chosen in the plane $Y=0$ where $\Omega=n \pi$ with $n=0,2,4, \ldots$ if $m>0$ and $n=1,3,5, \ldots$ if $m<0$. At these points $Z_{2}$ has its minimum value and makes the maximum excursion in $Z_{2}$ on a given orbit over a half-period. The composite quasi-phase-matching trajectory is constructed as before, changing the sign of the quadratic coefficients each time the plane $Y=0$ is crossed. In a system where the generated waves start from noise, those waves initially near the optimum relative phase grow most efficiently. In systems where the process is seeded, the relative phase is tuned to achieve optimum conversion.

Because the nonlinear period varies as the harmonic grows, optimizing the conversion efficiency requires that the length of the piecewise segments be varied along 
the propagation path. If $m$ is large enough, only a few layers are needed to produce complete conversion. The nonlinear periods are calculated using standard techniques (see also Alber, Luther, Marsden and Robbins [1998b] for optimization of the linear mismatch of averaged wave systems). If the length of the piecewise segments can not be varied, corrections to both $l_{c}$ and the initial relative phase give the constrained optimum conversion efficiency for the system. Note that for small $m$ the nonlinear period shifts are rather small.

Quasi-phase-matching is a robust technique. Note that if there are small errors in the the distance between each switch in the $\gamma_{j}$, they are not compounded directly and have no catastrophic effects.

The usual strategy for quasi-phase-matching described above is only one of many possibilities for controlling energy flow in wave interactions. Any two points on the three-wave surface can be connected by a composite $C^{0}$ trajectory if the system parameters are modulated between at least two states. In standard quasi-phasematching the two states are the two signs of $\gamma_{j}$. An alternate strategy for the robust control of frequency conversion at any value of $m$ works by modulating the sign of the mismatch parameter at a period shorter than the oscillation period for frequency conversion. The portions of the trajectories that are most nearly vertical produce the most conversion and are located near $X=0$. Therefore, in contrast to the standard quasi-phase-matching strategy, the optimum initial data has relative phase near $\Omega=n \pi / 2, n=1,2 \ldots$ Geometrically, the composite trajectory looks like a zig-zag stepping up the side of the three-wave surface along $X=0$. In Fig. 4.2 a trajectory is plotted on the three-wave surface.

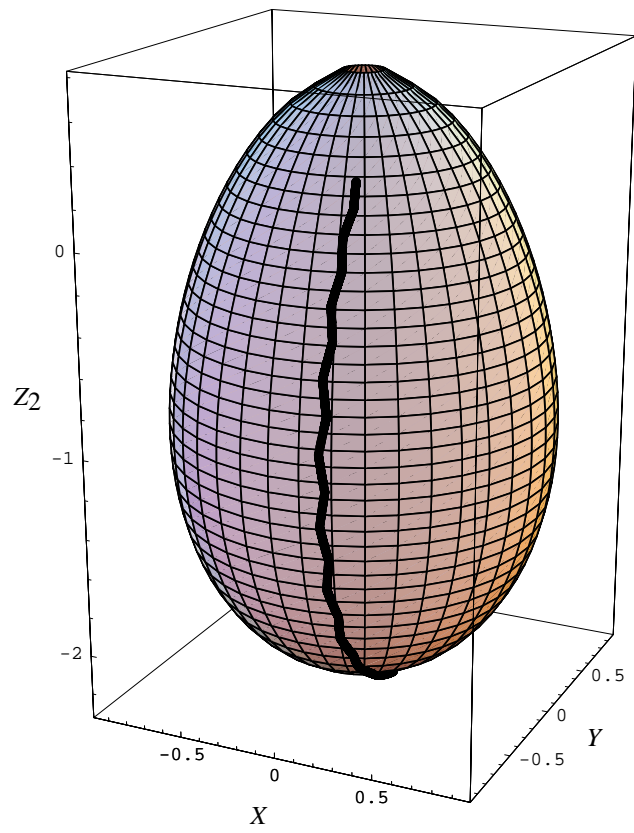

Figure 4.2: A composite trajectory for the zig-zag control. 
We refer to this strategy as the $\boldsymbol{z i g}$ - $\boldsymbol{z a g}$ control. In each step the trajectory goes less than half of the way around the three-wave surface. Notice that in general the length of the component trajectories is not critical. Small randomly distributed errors in the length from segment to segment tend not to cause early saturation of the conversion. As in conventional quasi-phase-matching, a relatively small amount of conversion is obtained between each modulation of the parameters, while the net conversion can be quite large.

This geometric approach to controlling wave interactions extends to a broad class of resonant wave interactions, and it introduces a general way to view nonlinear control strategies for nonlinear wave interactions. In nonlinear optics it underscores the idea that engineering dynamical systems can improve the net performance of optical materials.

\section{$5 \quad$ Projection of the Reduced Phase Space and Billiards}

The piecewise constant control strategies described above generate billiard trajectories on the three-wave surfaces. In this section, we elaborate on this construction. Using the piecewise constant controls described above billiard trajectories are generated by gluing together segments of solutions of different systems of three-wave equations; these systems differ from one another by the choice of coefficients $\gamma_{i}$ of the quadratic terms or $\Delta k$ of linear terms. To specify a particular strategy, the rules for changing these parameters and the time the system evolves between these changes must be specified. In what follows we discuss three examples.

In the first case we discuss, the three-wave system switches back and forth between two states defined by changing the quadratic coefficients after evolving for a full half-period. The second case is the same except that the time is defined by a coherence length (it could be longer or shorter than a full half period) and again we switch between two three-wave systems. In the third case we consider a billiard trajectory on the three-wave surface obtained by lifting a Birkhoff billiard trajectory inside the domain bounded by a meridian of the three-wave surface. In this case the switching times are determined by the reflection times, and the constants $\gamma_{i}$ characterizing a segment of the billiard trajectory after each reflection are calculated by lifting the billiard trajectory to a curve on the three-wave surface and identifying it with a curve that is the intersection of a plane and the three-wave surface. This plane defines a unique choice of constants for a three-wave system.

Half-period. Now we consider the first case mentioned above. Using the quasiphase-matching technique and taking the propagation time to be half the nonlinear period of each orbit, the boundary of the billiard is a meridian of the three-wave surface.

The invariant coordinates we choose to effect the reduction from $\mathbb{C}^{3}$ to the threewave surfaces in $\mathbb{R}^{3}$ are particularly useful here. Notice that the reduced Hamiltonian is independent of $Y$. When projected onto the $\left(X, Z_{2}\right)$-plane, trajectories on the three-wave surface become lines. Using the quasi-phase-matching control strategy where the signs of the quadratic coefficients are switched after one half period of the 
motion, trajectories starting at $Y=0$ end on $Y=0$. The curve $\phi\left(X, Y=0, Z_{2}\right)=0$, namely a meridian, is the boundary of the billiard in the $\left(X, Z_{2}\right)$-plane. The angle between incoming and outgoing segments of this billiard is the same for all points of reflection. These reflection conditions are illustrated in Fig. 5.1.

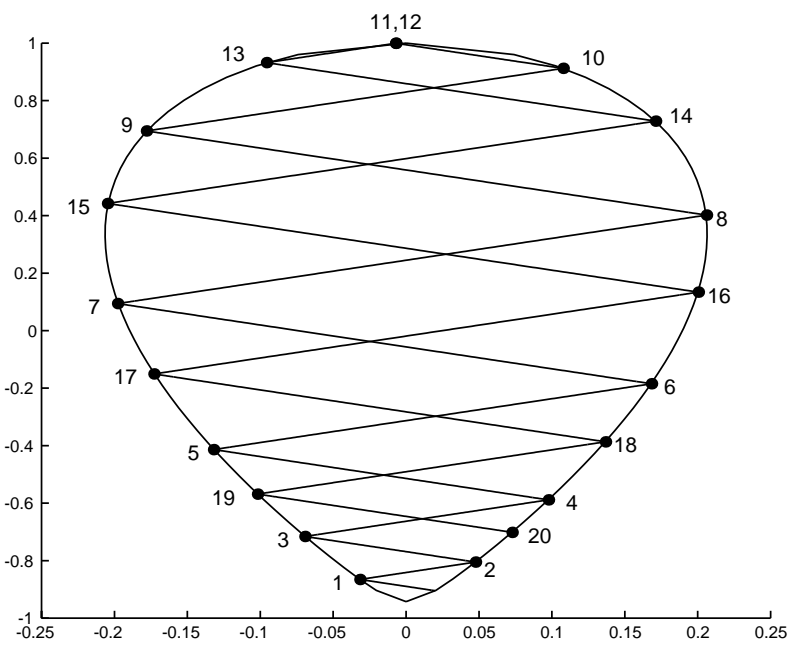

Figure 5.1: Billiard flow for quasi-phase-matched controls. The piece wise constant controls are switched between two states at half the nonlinear period. The locus of these points lie on the boundary $\phi\left(X, 0, Z_{2}\right)$ when projected into $\left(X, Z_{2}\right)$.

A formula for the $Z_{2}$-motion is obtained by reducing (3.7)-(3.9) to quadratures. One obtains the following potential equation,

$$
\frac{1}{2}\left(\frac{d Z_{2}}{d t}\right)^{2}=-2\left(s_{1} \gamma_{1}+s_{2} \gamma_{2}\right)\left[\phi\left(0,0, Z_{2}\right)-r Z_{2}\right]-\frac{\Delta k^{2} Z_{2}^{2}}{2}+C
$$

where

$$
r=\Delta k H_{r}+\Delta k^{2}\left(K_{1}+\frac{s_{2} \gamma_{2} K_{2}}{s_{2} \gamma_{2}+s_{3} \gamma_{3}}\right)
$$

is a constant and $X$ is obtained as a function of $Z_{2}$ through the reduced Hamiltonian $H_{r}$.

Fixed Coherence Length. The propagation length can be chosen to be a fixed distance, for instance the coherence length, $l_{c}=\pi / \Delta k$. An example is shown in the numerically generated plot in Fig. 5.2.

As was mentioned in the beginning of this section, in the most general case, billiards are constructed directly on the three-wave surface itself. As an example, consider the standard implementation of quasi-phase-matching control that uses a fixed propagation distance $l_{c}$ during each piece-wise constant segment of the control. (This distance as measured along the curve coincides with the evolution parameter $t$ 


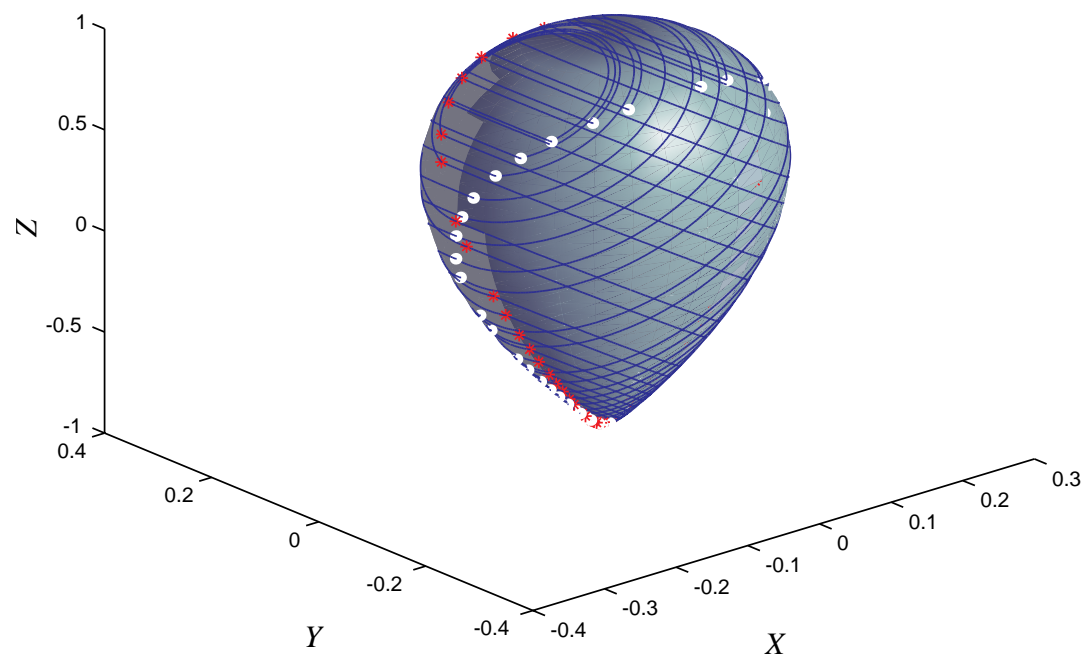

Figure 5.2: Billiard flow for quasi-phase-matched controls. The piece-wise constant controls are switched between two states at half the linear period as is typical in devices. The locus of these points appears to lie on two curves that intersect a finite number of times on the boundary $\phi\left(X, 0, Z_{2}\right)$.

or the geodesic length of the curve.) In what follows we calculate points of reflection of the billiard trajectory for a given initial point. Equation (5.1) yields the following integral problem of inversion

$$
\int_{Z_{2}^{0}}^{Z_{2}} \frac{d Z_{2}}{\sqrt{C_{ \pm}\left(Z_{2}\right)}}=t+t_{0}
$$

where $C_{ \pm}\left(Z_{2}\right)$ denotes a polynomial of the second order in $Z_{2}$ with + and - indicating the choice of the signs of $\gamma^{\prime} s$. Now, one can describe a particular billiard trajectory $\left(Z_{2}(0), Z_{2}(1), \ldots, Z_{2}(2 n)\right)$ by fixing initial point at $Z_{2}=Z_{2}(0)$ and by inverting the following elliptic integrals one by one:

$$
\begin{aligned}
& \int_{Z_{2}(2 k)}^{Z_{2}(2 k+1)} \frac{d Z_{2}}{\sqrt{C_{+}\left(Z_{2}\right)}}=(k+1) l_{c}+t_{0} \\
& \int_{Z_{2}(2 k+1)}^{Z_{2}(2 k+2)} \frac{d Z_{2}}{\sqrt{C_{-}\left(Z_{2}\right)}}=(k+2) l_{c}+t_{0}
\end{aligned}
$$

where $k=0, \ldots,(n-1)$.

One now wishes to find a curve on the three wave surface with the property that with initial conditions on the curve, a billiard trajectory has all its reflection points on a single pair of intersecting curves. The preceding formulas give an implicit relation that points on such a curve must satisfy. Figure 5.2 provides an example of 
such billiard trajectory together with a set of reflection points which appear to lie on two curves.

In the special case when the quadratic coefficient is switched at half the nonlinear period that was discussed above, this boundary is a single meridian curve. Considering ergodicity, periodic orbits, fixed points and stability in the context of these billiards and their generalizations will be the subject of future work.

Birkhoff Billiard. We now consider a further generalization of the billiard interpretation of control strategies on the three-wave surface by introducing the Birkhoff billiard. Here each segment of this Birkhoff billiard is mapped into a trajectory of a three-wave system by choosing parameter values for the control that produce the correct angle of reflection.

A standard class of Birkhoff billiards can be realized on the three-wave surfaces. They are constructed as follows. Consider a fixed domain either in the plane $\left(X, Z_{2}\right)$ (bounded by the projection $\phi\left(X, 0, Z_{2}\right)=0$ of the three-wave surface), or on the three-wave surface itself. Starting from the boundary of this region, evolve the three wave equations up to the next intersection with the billiard boundary. Impose the standard Birkhoff reflection condition using (5.1) and choose the value of the parameter $m$ that produces the correct angle of reflection. (The problem can be rescaled so that the three-wave surface is invariant with respect to changes in the magnitudes of the $\gamma_{k}$.) These conditions on the parameters of the three-wave equations provide an alternate strategy for controlling the three-wave system.

\section{Reconstruction and Phases}

Phase formulas for the three wave interaction can be developed that are somewhat parallel to the phase formulas for rigid body dynamics (see Marsden, Montgomery and Ratiu [1990] and Montgomery [1991]). The basic idea is to use a connection in the process of reconstructing the trajectories in the full space $\mathbb{C}^{3}$ from a knowledge of the trajectories in the reduced space (the three wave surfaces).

The reduced dynamics determines the evolution of the wave intensities. Once it has been solved, the full dynamics of the three-wave system, including the wave phases, may be reconstructed.

Here we only give the idea of what is involved in the reconstruction process. We consider the decay interaction, and, for definiteness, the particular case $\left(s_{1}, s_{2}, s_{3}\right)=$ $(1,-1,1)$, epitomized by $(1.1)-(1.3)$. We also assume that $\Delta k=0$; in this case the reduced dynamics is typically periodic (the exceptions are fixed points, homoclinic orbits and heteroclinic orbits), but the full dynamics is not. Thus, after a period $T$ of the reduced dynamics, the wave intensities return to their starting values while the phases are shifted.

The initial and final wave amplitudes are related by the phase symmetries (2.12)(2.13) (as remarked in Section 2, the third phase symmetry (2.14) is generated by 
the first two), so that

$$
\begin{aligned}
& q_{1}(T)=\exp \left(-i \Delta \phi_{1}\right) q_{1}(0) \\
& q_{2}(T)=\exp \left(-i \Delta \phi_{1}-i \Delta \phi_{2}\right) q_{2}(0) \\
& q_{3}(T)=\exp \left(-i \Delta \phi_{2}\right) q_{3}(0)
\end{aligned}
$$

There are two methods for calculating the total phase shifts $\Delta \phi_{1}$ and $\Delta \phi_{2}$. The first, the traditional method, involves integrating the system by means of actionangle variables. One finds a canonical transformation from the wave amplitudes $q_{j}$ to new canonical coordinates, in which two of the generalized momenta are the constants of motion $K_{1}$ and $K_{2}$; their conjugate angles, $\phi_{1}$ and $\phi_{2}$, are then ignorable coordinates. Once the reduced dynamics is known, the total phase shifts $\Delta \phi_{1}$ and $\Delta \phi_{2}$ may be computed by integrating Hamilton's equations

$$
\dot{\phi}_{j}=\frac{\partial H}{\partial K_{j}}
$$

in which the Hamiltonian is expressed in terms of action-angle variables, over the reduced period $T$.

While straightforward in principle, in execution the traditional method is rather involved. In contrast, the alternate method of geometric phases (Marsden, Montgomery and Ratiu [1990], Marsden [1992], Shapere and Wilczek [1989]), while requiring some additional theoretical machinery, leads in many cases to simpler calculations, as well as a suggestive geometric description of the phase shifts. Its application to the three-wave system may be viewed as a generalization of Montgomery's [1991] analysis of rigid body rotation. For discussions of the well known geometric phases which appear in polarization optics (e.g., Pancharatnam's phase), the reader is referred to Shapere and Wilczek [1989] and Bhandari [1997].

We shall not develop such formulas here, but rather refer to the author's paper Alber, Luther, Marsden and Robbins [1998a] for details. In the present paper we are focusing on control ideas that involve the reduced dynamics and not the phases, but in other contexts, control of the phases may be quite important. For instance, in nonlinear optics a phase shift of order $\pi$ enables all optical switching. The controls described here can be used to manipulate the optical wave interaction to reliably produce the desired phase shift. A second example is a laser in which the light is amplified through a three-wave mixing process. As light circulates in the optical cavity, its phase should be controlled to ensure that it is periodic over a round trip.

\section{The Lie-Poisson Formulation}

In this section the three-wave equations are written both on the dual of the Lie algebra of the group $\mathrm{SU}(3)$ or $\mathrm{SU}(2,1)$ using a Lie-Poisson structure; one can also formulate the problem on the Lie algebra using the Euler-Poincaré structure, focusing on variational principles, but we shall not undertake the latter here.

The Lie-Poisson description is obtained by recasting (1.1) as a differential equation in $\mathfrak{s u}(3)^{*}$, the dual of the Lie algebra of $\mathrm{SU}(3)$, for one of the decay interactions 
and a differential equation in $\mathfrak{s u}(2,1)^{*}$, the dual of the Lie algebra of $\mathrm{SU}(2,1)$, for the explosive interaction and the other two decay interactions.

Map to the Dual of the Lie Algebra. Define a map $U: \mathbb{C}^{3} \rightarrow \mathfrak{s u}(3)^{*}$ and a map $U: \mathbb{C}^{3} \rightarrow \mathfrak{s u}(2,1)^{*}$ as follows. Identify $\mathfrak{s u}(3)$ with $\mathfrak{s u}(3)^{*}$ using the standard Killing form:

$$
\langle A, B\rangle=\operatorname{Tr}(A B) .
$$

Thus, $\mathfrak{s u}(3)^{*} \cong \mathfrak{s u}(3)$ is concretely realized as the space of complex skew Hermitian matrices with zero trace. The standard Killing form is also used to pair $\mathfrak{s u}(2,1)$ with $\mathfrak{s u}(2,1)^{*}$. While the resulting inner product remains nondegenerate in this case, it does become Lorenzian.

To obtain complex Hamiltonian systems for which the complex conjugate equations are self consistent we restrict the map so that

$$
U=-M U^{\dagger} M^{-1}
$$

where $M=\operatorname{diag}\left(m_{1}, m_{2}, m_{3}\right)$ and $m_{j}= \pm 1$. Below we show that the $s_{j}$ are given by the $m_{j}$, so by choosing a set of values for the $m_{j}$, one fixes a particular three-wave system.

The map of $q=\left(q_{1}, q_{2}, q_{3}\right)$ to the matrix $U$ is then

$$
U=\left(\begin{array}{lll}
u_{1} & q_{1} & q_{2} \\
-\frac{m_{2}}{m_{1}} \bar{q}_{1} & u_{2} & q_{3} \\
-\frac{m_{3}}{m_{1}} \bar{q}_{2} & -\frac{m_{3}}{m_{2}} \bar{q}_{3} & u_{3}
\end{array}\right),
$$

where $U \in \mathfrak{s u}(3)^{*}$ for $\left(m_{1}, m_{2}, m_{3}\right)= \pm(1,1,1)$ and $U \in \mathfrak{s u}(2,1)^{*}$ otherwise. Here, the $u_{j}$ are purely imaginary to satisfy (7.2).

Define a second map $Q_{1}: \mathbb{C}^{3} \rightarrow \mathfrak{s u}(3)$ or $Q_{1}: \mathbb{C}^{3} \rightarrow \mathfrak{s u}(2,1)$ as

$$
Q_{1}=\left(\begin{array}{lll}
v_{1} & \alpha_{1} q_{1} & \alpha_{2} q_{2} \\
-\frac{m_{2}}{m_{1}} \alpha_{1} \bar{q}_{1} & v_{2} & \alpha_{3} q_{3} \\
-\frac{m_{3}}{m_{1}} \alpha_{2} \bar{q}_{2} & -\frac{m_{3}}{m_{2}} \alpha_{3} \bar{q}_{3} & v_{3}
\end{array}\right),
$$

where $\alpha_{j} \in \mathbb{R}$ are given in terms of the $\gamma_{j}$ as shown below and the $v_{j}$ are pure imaginary to satisfy (7.2).

We take $\alpha_{1}>\alpha_{2}>\alpha_{3}>0$ throughout. Below we show that the $u_{j}$ and $v_{j}$ can be chosen to produce the linear terms in $\Delta k$ in the three-wave equations.

With these definitions, the three-wave equations are written in matrix form as

$$
\frac{d U}{d t}=-\left[U, Q_{1}\right]
$$


where [,]: $\mathfrak{g} \times \mathfrak{g} \rightarrow \mathfrak{g}$ is the Lie bracket and in this context is equivalent to standard commutation of matrices. In component form these equations are:

$$
\begin{aligned}
\frac{d q_{1}}{d t} & =\left[\left(u_{2}-u_{1}\right) \alpha_{1}-\left(v_{2}-v_{1}\right)\right] q_{1}-\frac{m_{3}}{m_{2}}\left(\alpha_{2}-\alpha_{3}\right) q_{2} \bar{q}_{3}, \\
\frac{d q_{2}}{d t} & =\left[\left(u_{3}-u_{1}\right) \alpha_{2}-\left(v_{3}-v_{1}\right)\right] q_{2}-\left(\alpha_{3}-\alpha_{1}\right) q_{1} q_{3}, \\
\frac{d q_{3}}{d t} & =\left[\left(u_{3}-u_{2}\right) \alpha_{3}-\left(v_{3}-v_{2}\right)\right] q_{3}-\frac{m_{2}}{m_{1}}\left(\alpha_{1}-\alpha_{2}\right) \bar{q}_{1} q_{2} .
\end{aligned}
$$

By comparison with the three-wave equations (1.1)-(1.3), the linear coefficients become

$$
\begin{aligned}
& \left(u_{2}-u_{1}\right) \alpha_{1}-\left(v_{2}-v_{1}\right)=i \Delta k \\
& \left(u_{3}-u_{1}\right) \alpha_{2}-\left(v_{3}-v_{1}\right)=i \Delta k \\
& \left(u_{3}-u_{2}\right) \alpha_{3}-\left(v_{3}-v_{2}\right)=i \Delta k
\end{aligned}
$$

the nonlinear coefficients become

$$
\gamma_{1}=\left(\alpha_{2}-\alpha_{3}\right), \quad \gamma_{2}=\left(\alpha_{3}-\alpha_{1}\right), \quad \gamma_{3}=\left(\alpha_{1}-\alpha_{2}\right)
$$

and the signs are

$$
\left(s_{1}, s_{2}, s_{3}\right)=\left(\frac{m_{3}}{m_{2}},-1, \frac{m_{2}}{m_{1}}\right)
$$

With these identifications, one obtains the three-wave system (1.1)-(1.3) after $q_{k} \rightarrow$ $i q_{k}$.

Note that with this definition, $\sum \gamma_{k}=0$ automatically.

The Quadratic Invariants. The quadratic invariants (2.9)-(2.11) for (7.6)-(7.8) are

$$
\begin{aligned}
2 K_{1} & =\frac{m_{2}\left|q_{1}\right|^{2}}{m_{3}\left(\alpha_{2}-\alpha_{3}\right)}+\frac{\left|q_{2}\right|^{2}}{\left(\alpha_{1}-\alpha_{3}\right)}, \\
2 K_{2} & =\frac{\left|q_{2}\right|^{2}}{\left(\alpha_{1}-\alpha_{3}\right)}+\frac{m_{1}\left|q_{3}\right|^{2}}{m_{2}\left(\alpha_{1}-\alpha_{2}\right)}, \\
2 K_{3} & =\frac{m_{2}\left|q_{1}\right|^{2}}{m_{3}\left(\alpha_{2}-\alpha_{3}\right)}-\frac{m_{1}\left|q_{3}\right|^{2}}{m_{2}\left(\alpha_{1}-\alpha_{2}\right)} .
\end{aligned}
$$

If any two of these are positive or negative definite solutions are necessarily bounded. When this is not true solutions may blow up in finite time. If $m=\left(m_{1}, m_{2}, m_{3}\right)$ is $\pm(1,1,1), \pm(1,1,-1)$ or $\pm(1,-1,-1)$ the system corresponds to a decay interaction and if $m= \pm(1,-1,1)$ it is an explosive interaction. Notice that from the definition of the map $U$ one decay interaction is associated with $\mathfrak{s u}(3)$ and the other two as well as the explosive interaction are associated with $\mathfrak{s u}(2,1)$. 
The Quadratic Hamiltonian. The quadratic Lie-Poisson Hamiltonian is $\mathrm{H}_{2}=$ $-\operatorname{Tr}\left(U Q_{1}\right) / 2$, and it has the explicit form

$$
H_{2}=-\frac{1}{2} \sum_{k=1}^{3} u_{k} v_{k}+\frac{m_{2}}{m_{1}} \alpha_{1}\left|q_{1}\right|^{2}+\frac{m_{3}}{m_{1}} \alpha_{2}\left|q_{2}\right|^{2}+\frac{m_{3}}{m_{2}} \alpha_{3}\left|q_{3}\right|^{2},
$$

This Hamiltonian can be written in terms of the quadratic invariants as

$$
H_{2}=-\frac{1}{2} \sum_{k=1}^{3} u_{k} v_{k}+2 \alpha_{1}\left(\alpha_{2}-\alpha_{3}\right) \frac{m 3}{m_{1}}\left(K_{1}+\beta K_{2}\right),
$$

where

$$
\beta=\left(\frac{\alpha_{3}}{\alpha_{1}}\right)\left(\frac{\alpha_{1}-\alpha_{2}}{\alpha_{2}-\alpha_{3}}\right)
$$

The Lie-Poisson Bracket. As we show below, $Q_{1}=-\delta H_{2} / \delta U$, so we can write

$$
\frac{d U}{d t}=\left[\frac{\delta H_{2}}{\delta U}, U\right]
$$

The general theory of Lie-Poisson structures (see Marsden and Ratiu [1998]) is used to construct the Lie-Poisson bracket

$$
\{f, k\}_{1}(U)=-\left\langle U,\left[\frac{\delta f}{\delta U}, \frac{\delta k}{\delta U}\right]\right\rangle,
$$

where for $\mathfrak{g}=\mathfrak{s u}(3)$ or $\mathfrak{s u}(2,1), f, k: \mathfrak{g}^{*} \rightarrow \mathbb{R}, \delta f / \delta U, \delta k / \delta U \in \mathfrak{g}$, and $U \in \mathfrak{g}^{*}$.

Theorem 7.1 The Euler equation (7.5) governs the evolution of the matrix $U$ and is equivalent to the three-wave equations (1.1)-(1.3). This equation for $U$ is the Hamiltonian evolution equation associated with a non-canonical Hamiltonian structure having the (standard left invariant) Lie-Poisson bracket and a quadratic Hamiltonian. Realized through the evolution of $U$, we have the following:

1. the three-wave decay equations are Lie-Poisson equations on $\mathfrak{s u}(3)^{*}$ for $s=$ $(1,-1,1)$ and $m= \pm(1,1,1)$;

2. the three-wave decay equations are Lie-Poisson equations on $\mathfrak{s u}(2,1)^{*}$ for $s=$ $(-1,-1,1)$ and $m= \pm(1,1,-1)$, and also for $s=(1,-1,-1)$ and $m=$ $\pm(1,-1,-1)$;

3. the explosive three-wave equations are Lie-Poisson equations on $\mathfrak{s u}(2,1)^{*}$ for $s=(-1,-1,-1)$ and $m= \pm(1,-1,1)$.

Here we assume without loss of generality that $\gamma_{1}, \gamma_{3}>0$ and $\gamma_{2}<0$ (equivalently $\left.\alpha_{1}>\alpha_{2}>\alpha_{3}>0\right)$. 
Proof Let $F: \mathfrak{g}^{*} \rightarrow \mathbb{R}$, then with the definitions above, $d F / d t=\left\{F, H_{2}\right\}$, or

$$
\left\langle\frac{\delta F}{\delta U}, \frac{d U}{d t}\right\rangle=-\left\langle U,\left[\frac{\delta F}{\delta U}, \frac{\delta H_{2}}{\delta U}\right]\right\rangle
$$

where $\langle$,$\rangle is the trace defined above. Now,$

$$
D H_{2}(U) \cdot V=-\frac{1}{2} \operatorname{Tr}\left(V Q_{1}(U)\right)-\frac{1}{2} \operatorname{Tr}\left(U Q_{1}(V)\right),
$$

for $V \in \mathfrak{g}^{*}$. We claim that $Q_{1}$ is a symmetric linear function of $U$. In fact, one can check directly that $Q_{1}(U)_{i, j}=c_{i, j} U_{i, j}$ (no sum), where $c_{i, j}$ is a symmetric matrix. Thus,

$$
\operatorname{Tr}\left(U Q_{1}(V)\right)=\operatorname{Tr}\left(\sum_{j} U_{k, j} c_{j, k} V_{j, k}\right)=\sum_{j, k} U_{k, j} c_{j, k} V_{j, k}=\operatorname{Tr}\left(Q_{1}(U) V\right) .
$$

Hence, $D H_{2}(U) \cdot V=-\operatorname{Tr}\left(V Q_{1}(U)\right)$ and so $\delta H_{2} / \delta U=-Q_{1}(U)$. Using this fact, write

$$
\left\langle\frac{\delta F}{\delta U}, \frac{d U}{d t}\right\rangle=-\left\langle\frac{\delta F}{\delta U},\left[U, Q_{1}\right]\right\rangle
$$

to obtain

$$
\frac{d U}{d t}=-\left[U, Q_{1}\right]
$$

It is checked that these indeed are the three-wave equations.

Connections to the Rigid Body The appearance of the three-wave equations suggests that they should be related to the Euler equations for the free rigid body. In fact having put the three wave equations in the general context of Euler equations for Lie groups, this connection between the two systems can be illustrated easily. Begin by making the maps $U$ and $Q_{1}$ real. Then $U: \mathbb{R}^{3} \rightarrow \mathfrak{s o}^{*}(3)$ and $Q_{1}: \mathbb{R}^{3} \rightarrow \mathfrak{s o}^{*}(3)$. Renaming them $M$ and $-\Omega$, respectively,

$$
\frac{d M}{d t}=[M, \Omega]
$$

where $M$ is now identified as the body angular momentum and $\Omega$ as the body angular velocity. Using the recurrence relations where we assume all $Q_{j}$ are now real so that they also drop to $\mathfrak{s o}^{*}(3)$, it follows that $M=J \Omega+\Omega J$, were $Q_{0}=-J$ and $A=J^{2}$. With this additional relation we obtain the Euler-Arnol'd equations for the free rigid body. The Manakov equations are also easily produced here taking $Q^{(1)}=-\xi J-\Omega$ and $P=\xi J^{2}+M$ to obtain,

$$
\frac{d}{d t}\left(\xi J^{2}+M\right)=\left[\xi J^{2}+M, \xi J+\Omega\right] .
$$

Because the group $\mathrm{SO}(3)$ is a subgroup of $\mathrm{SU}(3)$, the free rigid body is contained within the three-wave interaction as a real subspace. Similarly, the extension of the three-wave system to $\mathfrak{s u}(N)^{*}$ contains Manakov's $N$-component rigid body on $\mathfrak{s o}(N)^{*}$. 


\section{Connections between the Two Hamiltonian Structures}

The three-wave equations have now been expressed using both the well known canonical Hamiltonian structure and the Lie-Poisson structure. In this section the relationship between them is discussed. A recursion relation is also produced and it is shown to be the same one obtained using the Lax approach.

The Second Hamiltonian Structure. Modify the Lie-Poisson bracket for the three-wave equations as follows:

$$
\{f, k\}_{0}(U)=-\left\langle U_{0},\left[\frac{\delta f}{\delta U}, \frac{\delta k}{\delta U}\right]\right\rangle
$$

in which the first matrix is fixed at $U_{0}$, where $U_{0} \in \mathfrak{s u}(3)^{*}$ or $U_{0} \in \mathfrak{s u}(2,1)^{*}$ is independent of $t$ and is to be specified. Taking $\delta f / \delta U$ and $\delta k / \delta U$ at $U$, this new bracket produces the equations of motion,

$$
\frac{d U}{d t}=\left[U_{0}, \frac{\delta k}{\delta U}\right]
$$

By choosing $U_{0}$ to be a constant diagonal matrix with $\operatorname{Tr}\left(U_{0}\right)=0$ and $k \propto H_{3}$, so that $\delta k / \delta U=Q_{2}, Q_{2}$ is quadratic in the $q_{i}$, we arrive at the three-wave equations. In this way the scaled canonical Hamiltonian structure is obtained directly from the Lie-Poisson bracket. Compatibility follows since this is a "translation of the argument" of the Lie-Poisson bracket, where $\{\}=,\{,\}_{1}(U)+\xi\{,\}_{0}\left(U_{0}\right)$ for an arbitrary real constant $\xi$. Both $\{,\}_{1}$ and $\{,\}_{0}$ are Poisson Brackets and the LiePoisson bracket with a shifted argument is also a Poisson bracket (see Arnol'd and Givental [1990], Trofimov and Fomenko [1994]). The two three-wave brackets are therefore compatible.

The Recursion Relation. Having obtained the Lie-Poisson structure and the compatibility of the two Poisson brackets the recursion relation for the three-wave equations are found. Equate the two Poisson brackets and write

$$
\left\langle U_{0},\left[\frac{\delta f}{\delta U},\left(\frac{\delta k_{j+1}}{\delta U}\right)\right]\right\rangle=\left\langle U,\left[\frac{\delta f}{\delta U},\left(\frac{\delta k_{j}}{\delta U}\right)\right]\right\rangle .
$$

For this relation to hold the Lie brackets,

$$
\left[\left(\frac{\delta k_{j+1}}{\delta U}\right), U_{0}\right]=\left[\left(\frac{\delta k_{j}}{\delta U}\right), U\right]
$$

must also be equal. This is exactly the recursion relation obtained using the Lax approach. For the three-wave system it is invertible, and a complete set of $(\delta k / \delta U)_{j}$ is constructed. 
The Lax Equations. To demonstrate the connection with the Lax approach let $D, P, Q \in \mathfrak{s u}(3)^{*}$ or let $D, P, Q \in \mathfrak{s u}(2,1)^{*}$. Write

$$
\begin{aligned}
\lambda D & =[P, D], \\
\frac{d D}{d t} & =[Q, D] .
\end{aligned}
$$

Compatibility of these two equations leads to

$$
\frac{d P}{d t}+[P, Q]=0
$$

Let

$$
P=\xi A+U \quad \text { and } \quad Q^{(N)}=\sum_{j=0}^{N} Q_{j} \xi^{N-j},
$$

where $A, U, Q_{j} \in \mathfrak{s u}(3)$ or $A, U, Q_{j} \in \mathfrak{s u}(2,1)$. Define $A$ to be $A=\operatorname{diag}\left(\beta_{1}, \beta_{2}, \beta_{3}\right)$ with $\sum_{k=1}^{3} \beta_{k}=0$. The $Q_{j}$ are general elements of the Lie algebra. As in (7.3), $U$ maps $\mathbb{C}^{3}$ into $\mathfrak{s u}(3)^{*}$ or $\mathfrak{s u}(2,1)^{*}$. With this definition for $P$, (8.7) becomes

$$
\frac{d U}{d t}+\xi\left[A, Q^{(N)}\right]+\left[U, Q^{(N)}\right]=0 .
$$

Now using the series for $Q^{(N)}$, the coefficients of powers of $\xi$ yield

$$
\begin{aligned}
\frac{d U}{d t}+\left[U, Q_{N}\right] & =0 \\
& \vdots \\
{\left[A, Q_{j}\right]+\left[U, Q_{j-1}\right] } & =0, \\
& \vdots \\
{\left[A, Q_{0}\right] } & =0 .
\end{aligned}
$$

The first equation is the integrable three-wave system. The second is the recursion relation. The final equation constrains the $Q_{j}$ so that $Q_{0} \in \operatorname{ker} \operatorname{ad}_{A}$. Letting $Q_{j}=(\delta k / \delta U)_{j}$ and $A=U_{0}$ this is exactly the recursion relation obtained using the method of Poisson pairs. The recursion relation implies that $\left[U, Q_{1}\right]=-\left[A, Q_{2}\right]$, so the three-wave equations are also written

$$
\frac{d U}{d t}=\left[A, Q_{2}\right]
$$

Now we compute the $Q_{j}$ directly from the recursion relation with

$$
Q_{0}=\operatorname{diag}\left(\beta_{1}^{0}, \beta_{2}^{0}, \beta_{3}^{0}\right) .
$$

We will find that the $\beta_{j}^{0}$ are directly related to the $\alpha_{j}$ and the $\gamma_{j}$. Carrying out the recursion (8.9)-(8.11) explicitly for the three-wave equations with $N=1$ it is found 
that

$$
Q_{1}=\left(\begin{array}{ccc}
v_{1} & \frac{\beta_{2}^{0}-\beta_{1}^{0}}{\beta_{2}-\beta_{1}} q_{1} & \frac{\beta_{3}^{0}-\beta_{1}^{0}}{\beta_{3}-\beta_{1}} q_{2} \\
-\frac{m_{2}}{m_{1}} \frac{\beta_{2}^{0}-\beta_{1}^{0}}{\beta_{2}-\beta_{1}} \bar{q}_{1} & v_{2} & \frac{\beta_{3}^{0}-\beta_{2}^{0}}{\beta_{3}-\beta_{2}} q_{3} \\
-\frac{m_{3}}{m_{1}} \frac{\beta_{3}^{0}-\beta_{1}^{0}}{\beta_{3}-\beta_{1}} \bar{q}_{2} & -\frac{m_{3}}{m_{2}} \frac{\beta_{3}^{0}-\beta_{2}^{0}}{\beta_{3}-\beta_{2}} \bar{q}_{3} & v_{3}
\end{array}\right) .
$$

This is written more compactly as

$$
\left(Q_{1}\right)_{i j}=\frac{\beta_{i}^{0}-\beta_{j}^{0}}{\beta_{i}-\beta_{j}} U_{i j}
$$

for $i \neq j$.

By direct comparison with $Q_{1}$ in (7.4) we find that $\alpha_{1}=\left(\beta_{2}^{0}-\beta_{1}^{0}\right) /\left(\beta_{2}-\beta_{1}\right)$, $\alpha_{2}=\left(\beta_{3}^{0}-\beta_{1}^{0}\right) /\left(\beta_{3}-\beta_{1}\right), \alpha_{3}=\left(\beta_{3}^{0}-\beta_{2}^{0}\right) /\left(\beta_{3}-\beta_{2}\right)$.

At the next iteration

$$
\left(Q_{2}\right)_{i k}=\sum_{j=1}^{3} \Gamma_{i j k} U_{i j} U_{j k}+\frac{1}{\beta_{i}-\beta_{k}}\left(U_{i j} Q_{1 j k}-Q_{1 i j} U_{j k}\right)
$$

where

$$
\Gamma_{i j k}=\frac{1}{\beta_{i}-\beta_{k}}\left(\frac{\beta_{i}^{0}-\beta_{j}^{0}}{\beta_{i}-\beta_{j}}-\frac{\beta_{j}^{0}-\beta_{k}^{0}}{\beta_{j}-\beta_{k}}\right) .
$$

Note that $\Gamma_{i j k}$ is invariant under all permutations of its indices so we write $\Gamma=\Gamma_{i j k}$ and

$$
Q_{2}=\Gamma\left(\begin{array}{ccc}
0 & -\frac{m_{3}}{m_{2}} q_{2} \bar{q}_{3} & q_{1} q_{3} \\
-\frac{m_{3}}{m_{1}} \bar{q}_{2} q_{3} & 0 & -\frac{m_{2}}{m_{1}} \bar{q}_{1} q_{2} \\
\frac{m_{3}}{m_{1}} \bar{q}_{1} \bar{q}_{3} & -\frac{m_{2}}{m_{1}} q_{1} \bar{q}_{2} & 0
\end{array}\right)+
$$

where

$$
\begin{aligned}
& \alpha_{12}=\frac{\alpha_{1}\left(u_{1}-u_{2}\right)+\left(v_{2}-v_{1}\right)}{\beta_{1}-\beta_{2}} \\
& \alpha_{13}=\frac{\alpha_{2}\left(u_{1}-u_{3}\right)+\left(v_{3}-v_{1}\right)}{\beta_{1}-\beta_{3}}
\end{aligned}
$$


and

$$
\alpha_{23}=\frac{\alpha_{3}\left(u_{2}-u_{3}\right)+\left(v_{3}-v_{2}\right)}{\beta_{2}-\beta_{3}}
$$

Note that $\left[U, Q_{2}\right]=0$, terminating the recursion.

With these definitions, $d U / d t=\left[A, Q_{2}\right]$ yields

$$
\begin{aligned}
\frac{d q_{1}}{d t} & =-\frac{m_{3}}{m_{2}}\left(\beta_{1}-\beta_{2}\right) \Gamma q_{2} \bar{q}_{3}+\left[\alpha_{1}\left(u_{1}-u_{2}\right)+\left(v_{2}-v_{1}\right)\right] q_{1}, \\
\frac{d q_{2}}{d t} & =-\left(\beta_{3}-\beta_{1}\right) \Gamma q_{1} q_{3}+\left[\alpha_{2}\left(u_{1}-u_{3}\right)+\left(v_{3}-v_{1}\right)\right] q_{2}, \\
\frac{d q_{3}}{d t} & =-\frac{m_{2}}{m_{1}}\left(\beta_{2}-\beta_{3}\right) \Gamma q_{2} \bar{q}_{1}+\left[\alpha_{3}\left(u_{2}-u_{3}\right)+\left(v_{3}-v_{2}\right)\right] q_{3},
\end{aligned}
$$

which are the three-wave equations in (7.6)-(7.8) since $\left(\beta_{1}-\beta_{2}\right) \Gamma=\left(\alpha_{2}-\alpha_{3}\right)$, $\left(\beta_{3}-\beta_{1}\right) \Gamma=\left(\alpha_{3}-\alpha_{1}\right)$, and $\left(\beta_{2}-\beta_{3}\right) \Gamma=\left(\alpha_{1}-\alpha_{2}\right)$. Here again there is freedom to choose the $u_{j}$ and $v_{j}$ from the definition of $U$ and $Q_{1}$ so that the linear terms correspond with the linear terms of the three-wave equations.

Conservation Laws and Hamiltonians. The $Q_{j}$ are gradients of Hamiltonian functions, and $Q_{j}=-\delta H_{j} / \delta U$, where the Hamiltonians

$$
H_{j+1}=-\operatorname{Tr}\left(U Q_{j}\right) /(j+1) .
$$

Here, $(j+1)$ is the highest power of $q_{k}$ in $H_{j+1}$. The cubic Hamiltonian defined here is proportional to the one associated with the scaled canonical structure from above. The quadratic Hamiltonian, $\mathrm{H}_{2}$, is associated with the Lie-Poisson structure.

These conserved quantities are found in a number of ways. The method of Poisson pairs produces invariants and their involutivity. The so called master conservation law is obtained by showing that the equation

$$
\left\langle U, \frac{d D}{d t}\right\rangle=\left\langle U,\left[Q^{(1)}, D\right]\right\rangle
$$

reduces to

$$
\frac{d}{d t}\langle D, U\rangle=\xi\left\langle D,\left[U, Q_{0}\right]\right\rangle
$$

Then using the recursion relation and in this case $D=Q^{(2)}$, one finds that

$$
\frac{d}{d t}\langle U, D\rangle=0
$$

In this way the Hamiltonians

$$
H_{2}=-\frac{1}{2}\left\langle Q_{1}, U\right\rangle, \quad H_{3}^{\prime}=-\frac{1}{3}\left\langle Q_{2}, U\right\rangle,
$$

are obtained, where $H_{3}^{\prime}=-2 i\left(m_{3} / m_{1}\right) \Gamma H_{3}$ if $q_{k} \rightarrow i q_{k}$. 


\section{Discussion}

Equations (8.5) and (8.6) provide alternate methods for solving the three-wave equations. They are used to construct the Lax pair of (8.7), which are linear equations for the evolution of an associated eigenfunction. Recall that as $D$ evolves, its determinant and the values of $\operatorname{Trace}\left(D^{k}\right)$ remain invariant. Since the coefficients of the spectral curve, namely

$$
\Gamma=\operatorname{Det}(D-y \mathrm{Id})=0,
$$

involve only these quantities, $\Gamma$ is also invariant.

By constructing the Baker-Akheizer functions of the associated linear spectral problem or by constructing new coordinates using $D$, algebraic-geometric methods can be applied to integrate the system in terms of theta functions on three-sheeted Riemann surfaces, where the genus of the resulting surface depends on the number of degrees of freedom present in the solution.

Finally, recall that (8.7) is the Lax equation for $P$. If $P$ and $Q=Q^{(1)}$ are linear in $\xi$ then (8.7) contains the three-wave equations, as shown above; (8.7) is then the so called $\lambda$-representation for the three-wave equations (see Manakov [1976], Novikov [1994]).

The three-wave system exhibits a rich Hamiltonian structure that has only been partially discussed here. Note for instance that this system can be expressed in terms of the $R$-matrix representation. Also note that the $\lambda$-representation for the threewave equations is a reduction of the loop algebra associated with $\mathfrak{s u}(3)$ or $\mathfrak{s u}(2,1)$. A more complete treatment of the general structure of integrable equations of this type is found for instance in Arnol'd and Givental [1990], Trofimov and Fomenko [1994], Arnol'd and Novikov, eds. [1994].

The family of $n$-wave interactions is connected to the groups $\mathrm{SU}(n)$ and $\mathrm{SU}(p, q)$. The structures described above for the three-wave example also follow for these higher-dimensional groups. Here integrability of the $n$-wave interaction on $\mathbb{C}^{n}$ is connected with the fact that there are a series of $\mathrm{U}(1)$ subgroups in $\mathrm{SU}(n)$ and $\mathrm{SU}(p, q)$ that reduce the equations on $\mathbb{C}^{n}$ to equations on surfaces in $\mathbb{R}^{3}$. In Kummer [1990] the resonant Hamiltonian system with $n$-frequencies was analyzed using the reduction procedure discussed here for the three-wave system. Using $n-1$ independent $S^{1}$ symmetries the $n$-wave system is ultimately reduced to quadratures.

Solutions of the three-wave system analyzed here are also traveling wave or stationary solutions of an integrable partial differential equation (for solution of the partial differential equation, see Zakharov and Manakov [1973, 1979], Ablowitz and Haberman [1975], Kaup [1976, 1981], Newell [1985], Ablowitz and Clarkson [1991]). In this sense the integrable structure outlined above generalizes to the structure of the partial differential equation. More generally, each integrable system of ordinary differential equations is associated with a hierarchy of evolution equations through (8.5)-(8.6) by letting $\lambda \rightarrow \partial / \partial x, d / d t \rightarrow \partial / \partial t$ and associating $D, P$, and $Q$ with an appropriate group. For instance, the three-wave system is realized as an integrable PDE and the ODE system (1.1) gives traveling wave solutions. Further, the threewave system is closely connected to the rigid body. The Euler equations on the 
real subspace formed by taking $\mathfrak{s u}^{*}(3) \rightarrow \mathfrak{s o}^{*}(3)$ will then have a related real partial differential equation for which the Euler equations are stationary or traveling wave solutions.

Acknowledgments. MSA was partially supported by NSF grants DMS 9626672 and 9508711. GGL gratefully acknowledges support from BRIMS, Hewlett-Packard Labs and from NSF DMS under grants 9626672 and 9508711. The research of JEM was partially supported by the National Science Foundation and the California Institute of Technology. JMR was partially supported by NSF grant DMS 9508711, NATO grant CRG 950897 and by the Department of Mathematics and the Center for Applied Mathematics, University of Notre Dame.

\section{References}

Ablowitz, M.J. and P.A. Clarkson [1991] Solitons, Nonlinear Evolution Equations and Inverse Scattering, Cambridge University Press, Cambridge.

Ablowitz, M.J. and R. Haberman [1975] Resonantly coupled nonlinear evolution equations, J. Math Phys. 16, 2301-2305.

Ablowitz, M.J. and H. Segur [1981] Solitons and the Inverse Scattering Transform, SIAM, Philadelphia.

Abraham, R. and J.E. Marsden [1978] Foundations of Mechanics. Second Edition, Addison-Wesley.

Akhmediev, N.N. and A. Ankiewicz [1997] Solitons, Chapman \& Hall, London.

Alber, M.S., G.G. Luther and J.E. Marsden [1997] Complex billiard Hamiltonian systems and nonlinear waves, in: A.S. Fokas and I.M. Gelfand, eds., Algebraic Aspects of Integrable Systems: In Memory of Irene Dorfman, Vol. 26 of Progress in Nonlinear Differential equations (Birkhäuser) 1-15.

Alber, M.S., G.G. Luther, J.E. Marsden and J.W. Robbins [1998a] Geometric phases, reduction and Lie-Poisson structure for the resonant three-wave interaction, Physica D, to appear.

Alber, M.S., G.G. Luther, J.E. Marsden and J.W. Robbins [1998b] Geometry and control of $\chi^{(2)}$ processes and the generalized Poincaré sphere, preprint.

Armstrong, J.A., N. Bloembergen, J. Ducuing and P.S. Pershan [1962] Interaction between light waves in a nonlinear dielectric, Phys. Rev. 127, 1918-1939.

Arnol'd, V. I. [1989] Mathematical Methods of Classical Mechanics. Second Edition Graduate Texts in Math 60, Springer-Verlag.

Arnol'd, V.I. and A.B. Givental [1990] Symplectic geometry, Encyclopedia of Math. Sci. 4, Springer-Verlag, 1-136. 
Arnol'd, V.I. and S.P. Novikov, eds. [1994] Dynamical systems VII, Encyclopedia of Math. Sci. 16, Springer-Verlag.

Bhandari, R. [1997] Polarization of light and topological phases, Phys. Rep. 281, $2-64$.

Born, M. and E. Wolf [1980] Principles of Optics, Pergamon, Oxford.

Cushman, R. and D. Rod [1982] Reduction of the semi-simple 1:1 resonance, Physica D 6, 105-112.

David, D. and D.D. Holm [1990] Multiple Lie-Poisson structures, reductions, and geometric phases for the Maxwell-Bloch travelling wave equations, J. Nonlinear Sci. 2, 241-262.

David, D., D.D. Holm and M. V. Tratnik [1989] Integrable and chaotic polarization dynamics in nonlinear optical beams, Physics Lett. A 137, 355-364.

Duistermaat, J.J. and G.J. Heckman [1982] On the variation in the cohomology of the symplectic form of the reduced phase space, Inv. Math. 69, 259-269, 72, $153-158$.

Fejer, M.M., G.A. Magel, D.H. Jundt and R.L. Byer [1992] Quasi-phase-matched second harmonic generation: tuning and tolerances, IEEE J. Quantum Electron. 28, 2631-2654.

Fordy, A.P. and D.D. Holm [1991] A tri-Hamiltonian formulation of the self-induced transparency equations, Phys. Let. A. 160, 143-148.

Guckenheimer, J. and A. Mahalov [1992] Resonant triad interactions in symmetric systems, Physica D 54, 267-310.

Haller, G. and S. Wiggins [1996] Geometry and chaos near resonant equilibria of 3-DOF Hamiltonian systems, Physica D 90, 319-365.

Kaup, D.J. [1976] The three-wave interaction-a nondispersive phenomenon, Stud. Appl. Math. 55, 9-44.

Kaup, D.J. [1981] The solution of the general initial value problem for the full three dimensional three-wave resonant interaction, Proc. Joint US-USSR Symposium on Soliton Theory, Kiev, 1979, V. E. Zakharov and S.V. Manakov, eds., (North-Holland, Amsterdam), 374-395.

Kaup, D.J., A.H. Reiman and A. Bers [1979] Space-time evolution of nonlinear three-wave interactions: I. interaction in a homogeneous medium, Rev. Mod. Phys. 51, 275-309.

Kirk, V., J.E. Marsden and M. Silber [1996] Branches of stable three-tori using Hamiltonian methods in Hopf bifurcation on a rhombic lattice, Dyn. and Stab. of Systems 11, 267-302. 
Knobloch, E., A. Mahalov and J.E. Marsden [1994] Normal Forms for threedimensional Parametric Instabilities in Ideal Hydrodynamics, Physica D 73, 49-81.

Kummer, M. [1975] An interaction of three resonant modes in a nonlinear lattice, J. Math. Anal. and Apps. 52, 64.

Kummer, M. [1990] On resonant classical hamiltonians with $n$ frequencies, J. Diff. Eqns. 83, 220-243.

Manakov, S.V. [1976] Note on the integration of Euler's equations of the dynamics of and n-dimensional rigid body, Funct. Anal and its Appl 10, 328-329.

Marsden, J.E. [1992], Lectures on Mechanics London Mathematical Society Lecture note series 174, Cambridge University Press.

Marsden, J.E. and T.S. Ratiu [1998] Introduction to Mechanics and Symmetry. Texts in Applied Mathematics, 17, Springer-Verlag, Second Edition.

Marsden, J.E. and A. Weinstein [1974] Reduction of symplectic manifolds with symmetry, Rep. Math. Phys. 5, 121-130.

Marsden, J.E., R. Montgomery and T.S. Ratiu [1990] Reduction, symmetry, and phases in mechanics, Memoirs AMS 436.

McKinstrie, C.J. [1988] Relativistic solitary-wave solutions of the beat-wave equations, Phys. Fluids 31, 288-297.

McKinstrie, C.J. and X.D. Cao [1993] The nonlinear detuning of three-wave interactions, J. Opt. Soc. Am. B 10, 898-912.

McKinstrie, C.J. and G.G Luther [1988] Solitary-wave solutions of the generalised three-wave and four-wave equations, Phys. Lett. A 127, 14-18.

McKinstrie, C.J., G.G Luther and S.H. Batha [1990] Signal enhancement of colinear four-wave mixing, J. Opt. Soc. Am. B 7, 340.

Montgomery, R. [1991] How much does a rigid body rotate? A Berry's phase from the $18^{\text {th }}$ century, Am. J. Phys. 59, 394-398.

Newell, A.C. [1985] Solitons in Mathematics and Physics, SIAM, Philadelphia.

Novikov, S.P. [1994] Solitons and Geometry, Academia Nazionale dei Lincei and the Scuola Normale Superiore, Press Syndicate of the University of Cambridge, Cambridge.

Rustagi, K.C., S.C. Mehendale and S. Menakshi [1982] Optical frequency conversion in quasi-phase-matched stacks of nonlinear crystals, IEEE J. Quantum Electron. QE-18, 1029. 
Shapere, A. and F. Wilczek, eds. [1989] Geometric Phases in Physics, World Scientific.

Sjamaar, R. and E. Lerman [1991] Stratified symplectic spaces and reduction, Ann. of Math. 134, 375-422.

Toronov, V.U. and V.L. Derbov [1998] Topological properties of laser phase, J. Optical Soc. B 15, 1282-1290.

Trillo, S., S. Wabnitz, R. Chisari and G. Cappellini [1992] Two-wave mixing in a quadratic nonlinear medium: bifurcations, spatial instabilities, and chaos, Opt. Lett. 17, 637.

Trofimov, V.V. and A.T. Fomenko [1994] Geometric and algebraic mechanisms of the integrability of Hamiltonian systems on homogeneous spaces and Lie algebras, Encyclopedia of Math. Sci. 16, Springer-Verlag, 261-333.

Whitham [1974] Linear and Nonlinear Waves, Wiley-Interscience.

Zakharov, V.E. and S.V. Manakov [1973] Resonant interaction of wave packets in nonlinear media, Sov. Phys. JETP Lett. 18, 243-245.

Zakharov, V.E. and S.V. Manakov [1979] Soliton Theory, in Physics Reviews Soviet Scientific Reviews, ed. I. M. Khalatnikov, Section A 1, 133-190. 